neutrophil elastase (NE), proteinase 3 (PR3), and cathepsin G (CG)) and myeloperoxidase (MPO) expression by qRT-PCR. $\mathrm{NE}^{-/} / \mathrm{PR}^{-/} / \mathrm{CG}^{-/-}$triple knockout mice and in vitro depletion of neutrophils approaches were performed to determine the role of NSPs and neutrophils in estrogen-mediated inflammatory responses. The splenic neutrophil and NSPs expression in lupus-prone MRL-lpr, $\mathrm{B} 6-l p r$ and $\mathrm{NZB} / \mathrm{W}_{\mathrm{F} 1}$ and their respective controls were also analysed.

Results Although oestrogen reduced total splenocytes number, it markedly increased the splenic neutrophil numbers, NSPs and MPO expression in B6 mice (Figure 1). Splenic neutrophils, NSPs and MPO were also significantly increased in MRL-lpr, B6-lpr and NZB/ $/ \mathrm{W}_{\mathrm{F} 1}$ mice (Figure 2). Despite of the critical role of NSPs and neutrophils in inflammation, depletion of NSPs in vivo did neither affect oestrogen's ability to increase in splenic neutrophils nor the induction of inflammatory mediators from ex vivo activated splenocytes, and depletion of splenic neutrophils in vitro had also no obvious effect on NSPs expression (due to the increase of NSPs in cells other than neutrophils) and LPS-induced IFNg and MCP-1 (Figure 3).

Conclusions Overall, we demonstrated a remarkable commonality with regards to the increase of neutrophils and NSPs in the spleens of autoimmune-prone mice and estrogen-treated B6 mice.

\section{EFFECT OF MICROPARTICLES DERIVED FROM PATIENTS WITH LUPUS ERYTHEMATOSUS SYSTEMIC (SLE) ON MODULATION OF MICRORNAS 146A AND 126, IN A MONOCYTE CELL LINE}

${ }^{1} \mathrm{G}$ Vasquez, ${ }^{1} \mathrm{U}$ Carmona-Perez ${ }^{*},{ }^{2} \mathrm{CH}$ Muñoz-Vahos, ${ }^{2} \mathrm{AL}$ Vanegas-Garcia, ${ }^{1} \mathrm{M}$ Rojas. ${ }^{1}$ Universidad de Antioquia, Grupo de Inmunología Celular e Inmunogenética GICIG. Instituto de Investigaciones Médicas- Facultad de Medicina-, Medellin, Colombia; ${ }^{2}$ Hospital Universitario San Vicente Fundación, Sección Reumatología, Medellin, Colombia

\subsection{6/lupus-2017-000215.113}

Background and aims Important miRNAs are involved in the modulation of immune functions and can be found in the intra- and extracellular environments, in circulation attached to RNA-binding molecules or packed in form of microparticles (MP). Given this, MP could have an important role in intercellular communication, modulation and expression of miRNAs in their target cells.

To establish whether MP of SLE patients can modulate the expression of miRNAs (miRNA 126, miRNA 146a) and their target molecule (interferon response factor 5, IRF5) in the monocyte cell line U937.

Methods MP obtained from serum of SLE and other autoinmune diseases (OAD) patients, and healthy controls were used as stimulus in the cell line U937 to evaluate their effect over: 1) The expression of membrane markers through flow cytometry, 2) content of miRNAs 126 and 146a through PCR and 3) expression of their target molecule, IRF5 by Western Blot. Results We observed, a decrease in HLA-DR, CD18, CD119 and an increase of IL-6 in U937 stimulated with MP from healthy controls, patients with active and inactive SLE, as well as patients with OAD. Additionally, a positive effect over the expression of miR126 and a negative effect over the expression of miR146a were observed. IRF5 as a target of miRNA146, did not change after MP treatment independent of MP origin.
Conclusions Our results suggested that MPs may have a regulatory effects, inducing a decreased expression of membrane molecules and miRNAs-146 levels, without effect in IRF5. In addition, MPs increased levels of cytokines and miRNA-126, latter is related with the demethylation.

\section{N-ACETYL-L-CYSTEINE (NAC) CONTROLS OSTEOCLASTOGENESIS THROUGH REGULATING TH17 DIFFERENTIATION AND RANKL PRODUCTION IN RHEUMATOID ARTHRITIS}

${ }^{1} \mathrm{HR}$ Kim*, ${ }^{2} \mathrm{BM}$ Kim, ${ }^{1} \mathrm{KA}$ Lee, ${ }^{1} \mathrm{SH}$ Lee, ${ }^{2} \mathrm{KW}$ Kim. ${ }^{1}$ Konkuk University Hospital, Rheumatology, Seoul, Republic of Korea; ${ }^{2}$ Seoul St. Mary's Hospital- College of MedicineThe Catholic University, Convergent Research Consortium for Immunologic disease, Seoul, Republic of Korea

\subsection{6/lupus-2017-000215.114}

Background and aims This study aimed to determine the regulatory role of N-Acetyl-l-cysteine (NAC), an antioxidant, in IL17-induced osteoclast differentiation in rheumatoid arthritis (RA).

Methods After RA synovial fibroblasts were stimulated by IL17 , the expression and production of RANKL was determined by real-time PCR and ELISA. Human peripheral blood monocytes were cultured with M-CSF, IL-17, RANKL, and/or various concentrations of NAC, followed by counting of the cells for tartrate-resistant acid phosphatase activity to determine osteoclast formation. Osteoclastogenesis was also determined after cocultures of IL-17-stimulated RA synovial fibroblasts, Th17 cells and various concentrations of NAC with monocytes. After human peripheral $\mathrm{CD}^{+} \mathrm{T}$ cells were cultured with NAC under Th17 condition, IL-17, IFN-g, IL-4, Foxp3, RANKL and IL-2 expression and production was determined by flow cytometry or ELISA.

Results When RA synovial fibroblasts were stimulated by IL17, IL-17 stimulated the production of RANKL, and NAC reduced the IL-17-induced RANKL production in a dosedependent manner. NAC decreased IL-17-activated phosphorylation of mTOR, JNK and IkB. When human peripheral blood $\mathrm{CD}_{14}{ }^{+}$monocytes were cultured with M-CSF and IL17 or RANKL, osteoclasts were differentiated, and NAC reduced the osteoclastogenesis. After human peripheral CD4 ${ }^{+}$ $\mathrm{T}$ cells were co-cultured with IL-17-pretreated RA synovial fibroblasts or Th17 cells, NAC reduced their osteoclastogenesis. Under Th17 polarising condition, NAC decreased Th17 cell differentiation and IL-17 and RANKL production.

Conclusions NAC inhibits the IL-17-induced RANKL production in RA synovial fibroblasts and IL-17-induced osteoclast differentiation. NAC also reduced Th17 polarisation. NAC could be a supplementary therapeutic option for inflammatory and bony destructive processes in RA.

\section{QUNANTITATIVE AND FUNCTIONAL EVALUATION OF PLASMA MICROPARTICLES IN SYSTEMIC LUPUS ERYTHEMATOSUS}

\begin{abstract}
${ }^{1,2}$ A Lateef* ${ }^{*}{ }^{1} \mathrm{~L}$ Shaffiee, ${ }^{3} \mathrm{GK}$ Gill, ${ }^{1} \mathrm{P}$ Cheung, ${ }^{4} \mathrm{YC}$ Lim. ${ }^{1}$ National University Hospital, Medicine, Singapore, Singapore; ${ }^{2}$ Yong Loo Lin School of Medicine- National University of Singapore, Medicine, Singapore, Singapore; ${ }^{3}$ National University of Singapore, Physiology, Singapore, Singapore; ${ }^{4}$ National University of Singapore, Pathology, Singapore, Singapore
\end{abstract}

10.1136/lupus-2017-000215.115 
A

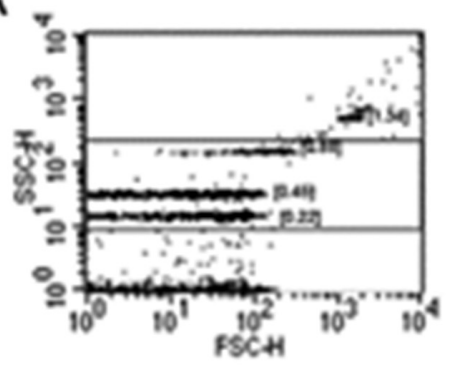

B

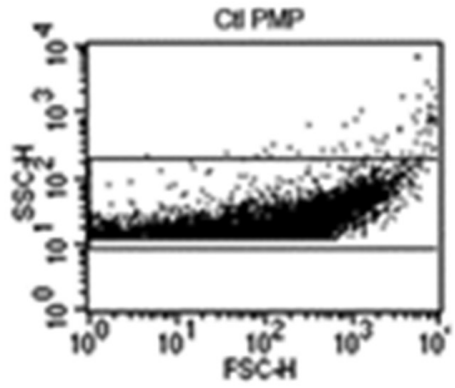

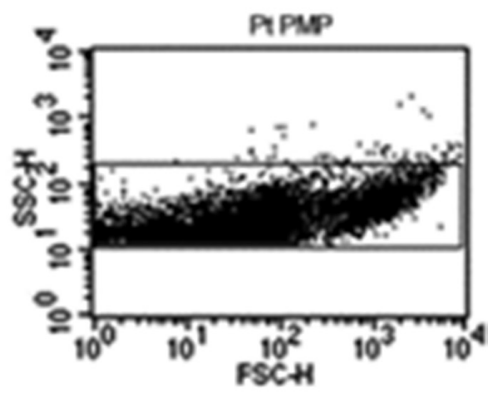

C
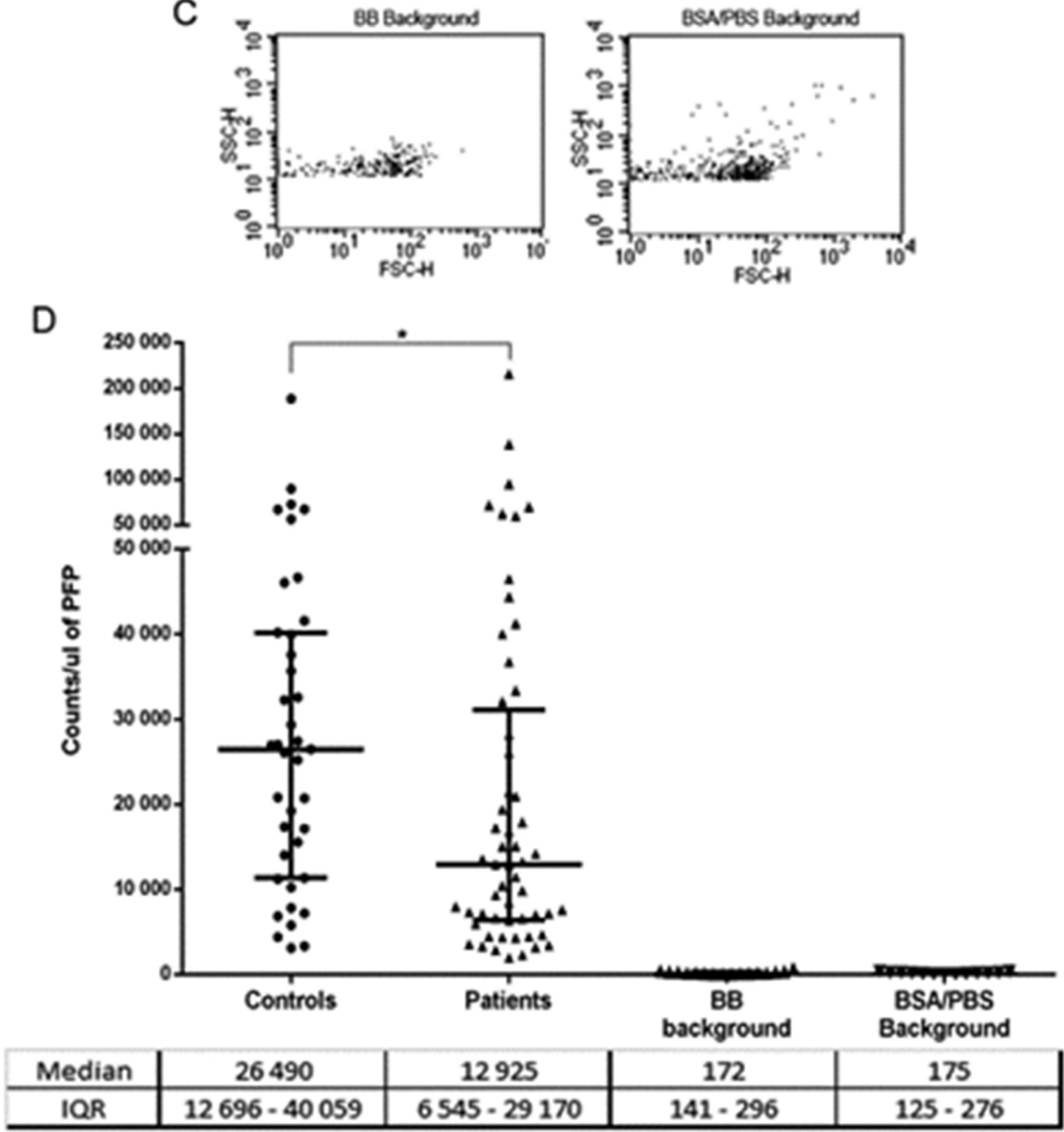

Abstract 115 Figure 1 SLE samples have lower PMPs than control samples. (A) PMP gate setup using 0.22, 0.45, 0.88 and 1.34 $\mu \mathrm{m}$ fluorescent polystyrene beads. (B) A representative example of control PMP (left) and patient PMP (right) diluted 1:10. Events falling outside the gate were not considered as PMP.Thresold foe PMP collection set according to SSC. (C) Time-matched backgrounds of the diluents binding buffer (BB, left) and 1\% bovine serum albumin dilutedin phosphate buffered solution (BSA/PBS, right). (D)PMP counts for control samples $(n=39)$, patient samples $(n=56)$, BB background $(n=16)$ and BSA/PBS background $(n=13)$. Controls had significantly higher PMP counts than patients $(p=0.01)$. Each data point shown represents 1 unique control or patient sample. Bars represent median with interquartile range. 

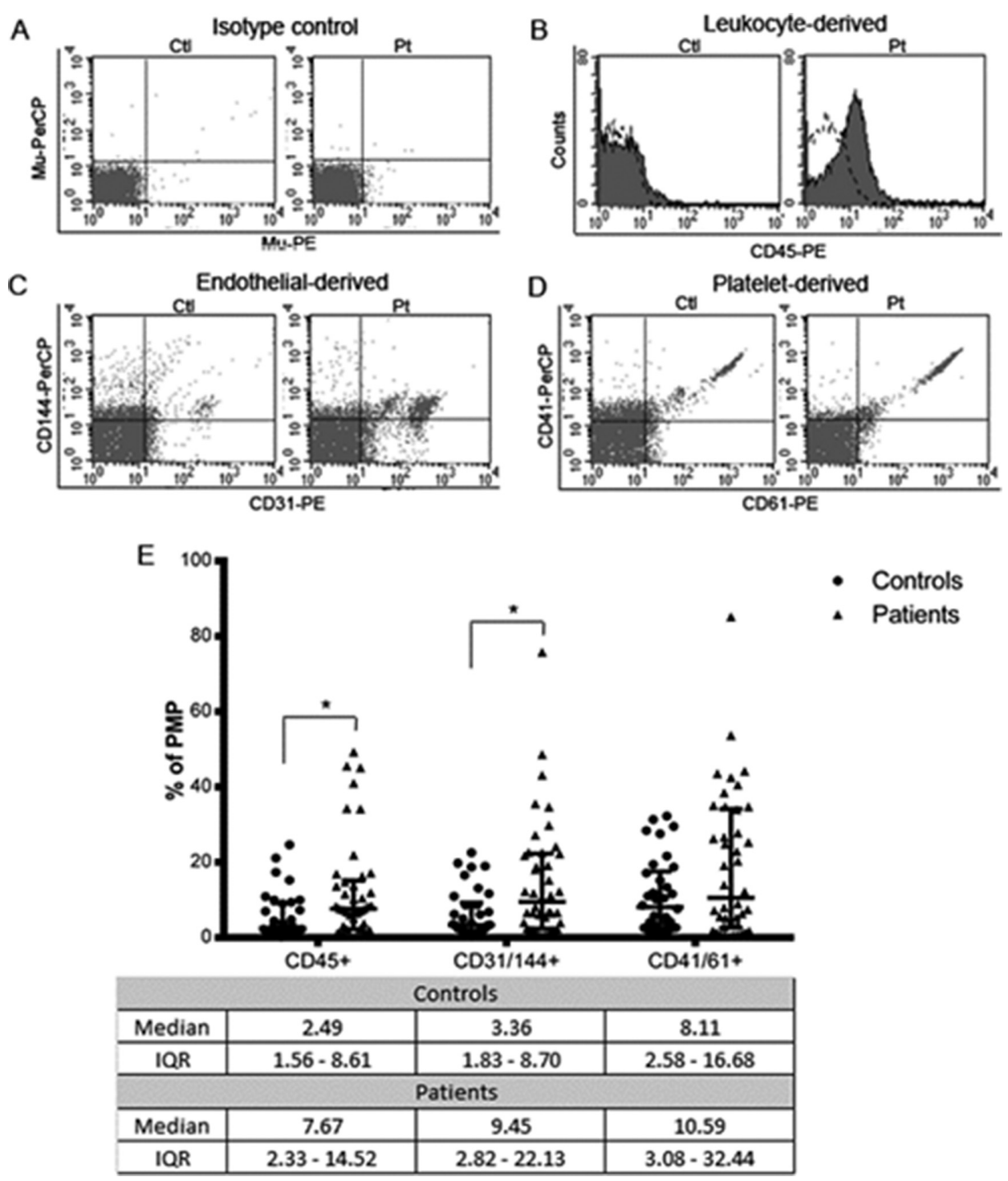

Abstract 115 Figure 2 Highewr percentage of leukocyte and edothelial derived PMP, But not platelet-derived PMP, are detected in SLE patients. (A) Isotype control for PE and PerCP-conjugated antibodies in representative control (left) and patient (right) samples. (B) Representative examples of CD45-PE positive staining in control (left) and patient (right) samples. (C) CD31-PE and CD144-PerCP double staining in a control (left) and a patient (right) Sample. (D) CD61-PE and CD41-PerCP double staining in a control (left)and a patient (right) sample. (E) Each data point shown represents 1 control or patient sample.Bars represent median and interquartile range. Significant differences between control $(n=34)$ and patient $(n=42)$ groups in the population of leukocyte $(p=0.02)$ and endothelial $(p=0.01)$ derived PMP using Mann-Whitney test.

Background and Aims Plasma microparticles (PMPs) are small $(0.1$ to $1.0 \mu \mathrm{m})$ membrane bound vesicles, released from plasma membrane during cell activation and apoptosis. The cytoplasmic and surface contents of PMPs vary according to the parental cell's lineage and physiological state. Altered numbers and profiles have been associated with thrombotic and inflammatory disorders including rheumatic diseases.

There have been variable reports on PMPs levels and profiles in Systemic Lupus Erythematosus (SLE). Some studies have reported higher levels while others showed similar or lower numbers with altered profiles. We compared PMPs in a multi-ethnic SLE cohort with a healthy control group, and explored their role in pathophysiology of SLE

Methods Clinical data and blood samples were collected from 56 consented SLE patients (median 45 years, 95\% females) who fulfilled the ACR criteria, and 39 healthy adults. PMP pellet was obtained by high speed centrifugation. Flow cytometry was used to enumerate and profile the PMPs, utilising fluorescent polystyrene nanobeads, lipophilic dyes and fluorescent-labelled antibodies. HL60, a promyelocytic cell line, was utilised to study functional effects of PMPs in a smaller subgroup of samples (8 from each arm).

Results SLE patients have lower PMP numbers compared to healthy controls (median: 12925 vs 26490 respectively, $\mathrm{p}=0.01$, Figure 1). SLE PMPs also exhibited significantly higher proportions of leukocyte, and endothelial markers (Figure 2). SLE PMPs induced significantly higher p38 phosphorylation in HL60 cells compared to control PMPs $(p=0.04$, Figure 3)

Conclusions Our data suggest that there are quantitative and functional differences in PMPs between SLE patients and healthy controls 